\title{
La presión positiva continua en la vía aérea mejora la somnolencia en pacientes con apneas obstructivas del sueño
}

Continuous positive airway therapy for treating sleepiness in a diverse population with obstructive sleep apnea. Patel S, White , Malhotra A, Stanchina M, Ayas N. Arch Intern Med. 2003; 163: 565-571.

\section{Objetivo}

Evaluar la utilidad de la terapia con presión positiva continua en la vía aérea (CPAP) para mejorar la somnolencia diurna en pacientes con síndrome de apnea del sueño obstructiva (ASO).

\section{Fuente y selección de los datos}

MEDLINE (1966 a 2001) bibliografía de articulos, metanálisis previos y revisiones; búsqueda en la base de datos Cochrane y consulta a expertos. Fueron seleccionados ensayos clínicos con algún grupo control que evaluaran la utilidad de la CPAP durante más de una semana en adultos con ASO y utilizaran, por lo menos, alguna forma estandarizada de evaluación de la somnolencia (Ver cuadro 1). El grupo control podía consistir en:ausencia de tratamiento, medidas generales (bajar de peso, reducir la ingesta de alcohol y sedantes, etc.) o placebo (ej.indicación de una píldora). Fueron excluidos los estudios que compararon CPAP con otros dispositivos. Dos investigadores calificaron en forma independiente a los artículos con la escala de Jadad* (calidad de la aleatorización, doble ciego, inclusión de los datos de las pérdidas) con buen acuerdo entre ambos.

Cuadro 1:formas validadas de evaluación de la somnolencia.

Escala de somnolencia de Epworth: utiliza ocho preguntas que evalúan la probabilidad de quedarse dormido en distintas situaciones. El puntaje va de 0 a 24 y se considera somnolencia significativa a un puntaje de 11 ó más.

Pruebas objetivas: se registra cuantos minutos tarda el paciente tratando de dormirse en una habitación oscura (promedio de cinco intentos) o cuanto logra mantenerse despierto en las mismas condiciones.

\section{Resultados principales}

Fueron incluidos 12 estudios. Considerando globalmente a todos los estudios, la reducción promedio en la escala de somnolencia fue de
2,94 puntos (IC95:1,61 a 4,26). Se observó una alta heterogeneidad* $^{*}$ entre los distintos estudios incluidos, que no pudo ser explicada por su diferente calidad ni por la naturaleza de la CPAP o del placebo utilizado;así como tampoco por el sexo, la edad o la proveniencia de los participantes. Cuando se incluyeron en el metanálisis sólo los trabajos que habían incluido a los pacientes más severos (más de 30 eventos de ASO por hora y más de 11 puntos en el puntaje de somnolencia) la mejoría fue de 4,75 puntos (IC95:2,97 a 6,53).Ver figura 1.

Figura 1:metanálisis de los seis trabajos que habían incluido a los pacientes más severos.

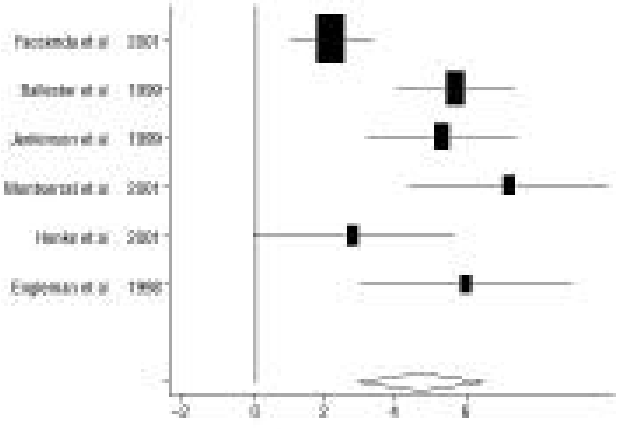

Se halló una diferencia de un minuto en el tiempo a quedarse dormido, estadísticamente significativa (aunque poco relevante clínicamente).

\section{Conclusiones}

El tratamiento con CPAP de los pacientes con apneas del sueño es efectivo para reducir la somnolencia diurna, con mayor beneficio en los pacientes más severos.

Fuente de financiamiento:Subsidios del National Institute of Health, Bethesda Md, Medical Research Council of Canada, y American Heart Association. EE.UU.

\section{Comentario}

Uno de cada cinco adultos de edad media ronca, y la prevalencia de ASO es de $3 \%$ en varones y de $2 \%$ en mujeres ${ }^{1}$. Casi el $80 \%$ de los pacientes son varones y la mayoría, obesos (más de la mitad tiene BMI mayor a 30 ).El $95 \%$ ronca y el $90 \%$ presenta una excesiva somnolencia diurna².

La disminución del calibre de la vía aérea superior durante el sueño genera un mecanismo de despertar que aumenta el tono de la faringe y le devuelve la permeabilidad.Puede ocurrir cientos de veces durante la noche y se acompaña de un aumento del tono simpático, lo que explicaría la asociación de este síndrome con hipertensión arterial y mayor riesgo cardiovascular.Esta alteración del sueño causa somnolencia diurna con impacto negativo en la calidad de vida, ineficiencia laboral y mayor riesgo de accidentes de tránsito.

Ante la sospecha de este síndrome se recomienda pesquisar somnolencia diurna excesiva sin causa clara (ej.deprivación del sueño, consumo de benzodiacepinas, ingesta excesiva de alcohol, retención de $\mathrm{CO} 2$ en las excacerbaciones de la enfermedad obstructiva crónica) y si su compañero de cama ha percibido que el paciente detenga su respiración durante el ronquido; estudiando con polisomnografía a los pacientes con alguno de los dos hallazgos positivos.Además de las medidas generales' (tratamiento de la obesi- dad y abandono del tabaco, sueño en decúbito lateral y reducción del consumo de alcohol y sedantes) a los pacientes con evidencia polisomnográfica de ASO se les puede ofrecer el uso de CPAP y/o corrección quirúrgica de la anomalía anatómica que pudieran tener ${ }^{3}$, con el objetivo de mejorar su calidad de vida y su productividad, reducir el riesgo de accidentes y disminuir la morbimortalidad cardiovascular.

Este metanálisis contesta parcialmente uno de los interrogantes que estaban poco claros todavía:la utilidad de la CPAP para mejorar la somnolencia. Destacamos que incluyó estudios sobre poblaciones diversas, lo que mejora la validez externa*. Pareciera haber cierto reaseguro contra el sesgo de publicación* ya que no hubo predominio de efecto positivo en ensayos de menos pacientes, ni cambios importantes de los resultados al excluir los ensayos más pequeños. Entre sus debilidades mencionamos que el mayor seguimiento fue 24 semanas (la mayoría de los trabajos duro cuatro a seis) y que la gran heterogeneidad ${ }^{*}$ de los resultados de los distintos estudios incluidos conspira contra de la validez de generar una medida sumaria.

Conclusiones de los comentadores: El tratamiento con CPAP de los pacientes con apneas del sueño es efectivo para reducir la somnolencia diurna en los pacientes más severos.

\section{Dr. Sergio Terrasa [ Unidad de Medicina Familiar y Preventiva del Hospital Italiano de Buenos Aires ]}

\section{Referencias}

1- Laitinen LA, Anttalainen U, Pietinalho A, Hamalainen P, Koskela K; Expert Advisory Group Listed in Foreword. Sleep apnoea:Finnish National guidelines for prevention and treatment 2002-2012.Respir Med.2003 Apr;97(4):337-65.

2- Str yjewski M. Enfoque del paciente roncador. En: Rubinstein A, Terrasa S, Durante E, Rubinstein E, Carrete P, Zárate M editores.Medicina Familiar y Práctica Ambulatoria. 2001, Buenos Aires. Editorial Médica Panamericana. Capítulo 93.Pagina 656-660.

2001, Buenos Aires. Editorial Médica Panamericana. Capítulo 93.Pagina 656-660. Med. 2000 Aug;64(8):465-8. 